\title{
Proton Pump Inhibitor Use Is Associated With an Increased Frequency of Hospitalization in Patients With Cystic Fibrosis
}

\author{
Fares Ayoub $^{\mathrm{a}, \mathrm{d}}$, Jorge Lascano ${ }^{\mathrm{b}}$, Giuseppe Morelli ${ }^{\mathrm{c}}$
}

\begin{abstract}
Background: Proton pump inhibitors (PPIs) are among the most commonly prescribed medications in clinical practice. PPI use has been associated with the development of community-acquired pneumonia. With a reported prevalence of gastroesophageal reflux disease (GERD) and PPI use that is higher than the general population, patients with cystic fibrosis (CF) are particularly vulnerable to PPI adverse effects. We sought to explore whether PPI use was associated with a higher number of hospitalizations for CF pulmonary exacerbation.
\end{abstract}

Methods: We conducted a longitudinal retrospective review in an academic outpatient setting. Patients $>18$ years of age with a diagnosis of $\mathrm{CF}$ and at least 1 year of follow-up were eligible for inclusion. Baseline characteristics, PPI use, and details of hospitalization through 1 year of follow-up were collected.

Results: One hundred fourteen patients met inclusion criteria. Fiftynine patients $(51.7 \%)$ were hospitalized at least once in the follow-up year, mean number of hospitalizations was $2.17( \pm 1.9)$. At least 6 months of PPI use was observed in 59 patients (51.7\%). In univariate analysis, PPI use was associated with a significantly higher mean number of hospitalizations ( 0.9 vs. $1.4, \mathrm{P}=0.009)$. In a multi-variable regression model, PPI use remained significantly associated with a higher number of hospitalizations $(\mathrm{P}=0.03)$, while controlling for risk factors traditionally associated with increased pulmonary exacerbations.

Conclusion: PPI use is highly prevalent in CF patients. Exposure to PPI therapy is independently associated with a higher number of hospitalizations for pulmonary exacerbation in CF patients.

Keywords: Proton pump inhibitors; Cystic fibrosis; Hospitalization; Community-acquired infections; Pneumonia

Manuscript submitted October 4, 2017, accepted October 12, 2017

aDepartment of Medicine, University of Florida, Gainesville, FL 32608, USA bAdult Cystic Fibrosis Center, Division of Pulmonary, Critical Care \& Sleep Medicine, University of Florida, Gainesville, FL 32608, USA

'Division of Gastroenterology, Hepatology \& Nutrition, University of Florida, Gainesville, FL 32608, USA

${ }^{\mathrm{d} C}$ Corresponding Author: Department of Medicine, University of Florida, Gainesville, FL 32608, USA. Email: fares.ayoub@medicine.ufl.edu

doi: https://doi.org/10.14740/gr917w

\section{Introduction}

Cystic fibrosis (CF) is the most common lethal genetic disease in Caucasians with an estimated 30,000 affected individuals in the United States alone [1]. A significant proportion of the morbidity and mortality in CF patients results from pulmonary exacerbations [2-4]. Pulmonary exacerbations have been linked to an irreversible decline in pulmonary function [5], a significant impact on health-related quality of life [6] and decreased survival [7]. Identifying modifiable risk factors for pulmonary exacerbations would lead to considerable benefits for CF patients.

Long-term proton pump inhibitor (PPI) use is prevalent in the CF population $[8,9]$. The majority of PPI use is for management of gastroesophageal reflux (GER), with a prevalence of $35-81 \%$ that is considered to be higher than the general population [8]. Studies in CF patients have found associations between GER and poor pulmonary function [10] and aggressive management of GER with PPI has been advocated by some experts [11]. However, whether PPI therapy alters pulmonary outcomes is unclear. Despite PPI therapy, risk of bile acid aspiration persists [12] and non-acid reflux has been found to be negatively correlated with pulmonary function [13].

PPI therapy has been linked to the development of community-acquired pneumonia (CAP) in both adults and children $[14,15]$. This association is thought to be due to an increase in intra-gastric $\mathrm{pH}$ leading to insufficient elimination and increased colonization of ingested pathogens, as well as direct inhibition of several leukocyte functions by gastric acid inhibitors $[16,17]$.

We sought to study the association between PPI use and rates of hospitalization for CF pulmonary exacerbations.

\section{Methods}

Our study was a longitudinal retrospective review of hospitalizations for CF pulmonary exacerbations over 1 year. Patients with CF seen at the University of Florida Adult Cystic Fibrosis Center between January and December 2016 were eligible for inclusion. Hospitalizations were assessed for the full year preceding the patient's index CF clinic visit in 2016. Inclusion criteria were age $>18$ years, a confirmed diagnosis of $\mathrm{CF}$ and at least 12 months of complete follow-up prior to the patient's index adult CF center visit in 2016. Patients with incomplete data, new patient referrals to the CF center during 2016 or 


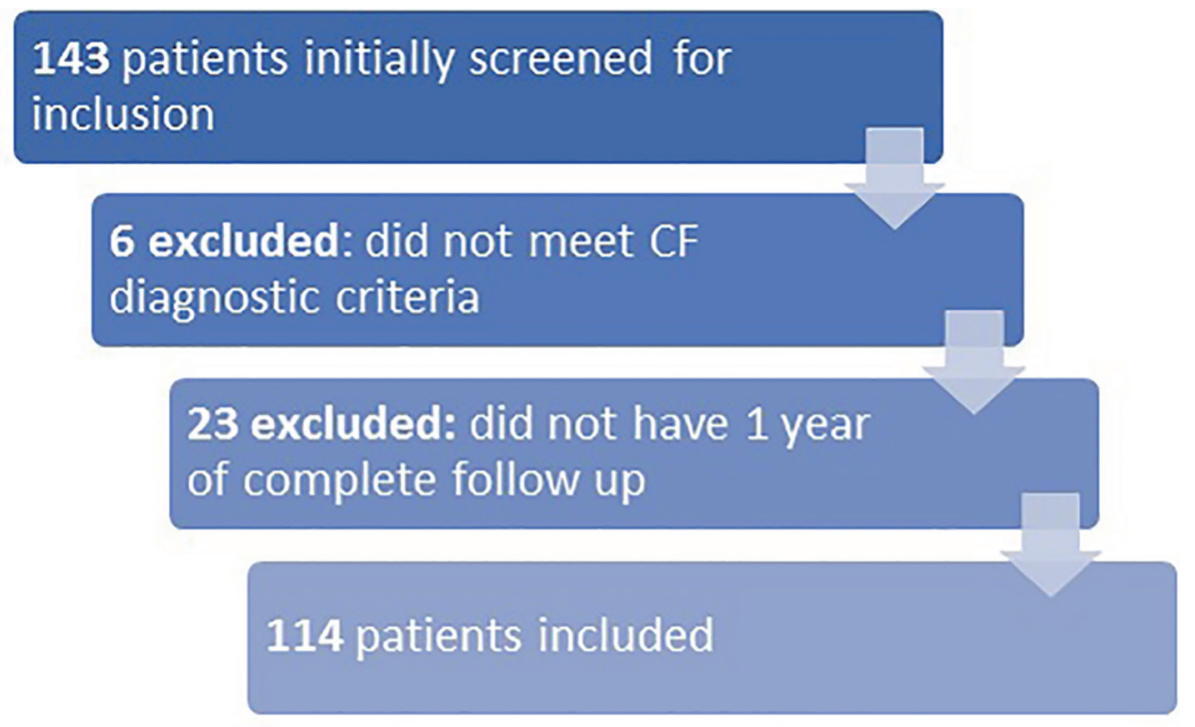

Figure 1. Flowchart of initial screening for eligibility, exclusion and final number of patients included in analysis.

those not meeting inclusion criteria above were excluded.

Data collected included age, gender, body mass index (BMI), genetic mutation analysis, percentage of predicted forced expiratory volume in 1 second $\left(\mathrm{ppFEV}_{1}\right)$, evidence of chronic pseudomonas colonization, history of pancreatic insufficiency and history of GER, PPI use prior to study year, type, dosage of PPI and number of hospitalizations for pulmonary exacerbation in the year of follow-up. Hospitalization for pulmonary exacerbation was defined as an inpatient admission where history, physical examination and/or imaging lead to the diagnosis of a pulmonary exacerbation requiring the use of intravenous antibiotics as determined by the admitting physician.

Diagnosis of CF was confirmed by the combination of clinical symptoms of $\mathrm{CF}$ and an elevated sweat chloride $\geq 60$ $\mathrm{mmol} / \mathrm{L}$ or the presence of two disease-causing mutations in CF transmembrane conductance regulator gene (CFTR). PPI use was defined as prescription and patient reported use of PPI for at least 6 months prior to year of study and continued during the year of study. Only those initiated on PPI therapy prior to the year of study were considered for inclusion in the PPI user arm, and this was to avoid inclusion of those initiated on PPI therapy during hospitalization and continued on such therapy after discharge, confounding our results. Patients newly initiated on PPI therapy during the year of observation or noted to use PPI as needed were not eligible for inclusion in either arm.

Diagnosis of GER was based on clinical symptoms and documentation in the medical record as no diagnostic testing of GER was available in most patients. Chronic pseudomonas colonization was defined as detection within a period of 6 months of a minimum of three positive Pseudomonas aeruginosa cultures, with at least 1 month between the positive cultures [18]. Patients were considered pancreatic insufficient if they demonstrated clinical symptoms and fecal elastase values less than $200 \mu \mathrm{g} / \mathrm{g}$ [19]. The study was approved by the institutional review board at the University of Florida College of Medicine.

\section{Statistical analysis}

Data are presented as proportions (mean $\pm \mathrm{SD}$ ) and for variables not conforming to a normal distribution as median and interquartile range (IQR). Two-sample comparisons were by Chi-square tests for proportions, Student's $t$-test for normally distributed variables, and Mann-Whitney U test for other variables. Shapiro-Wilk test was used to determine normality of continuous variables.

Due to the large number of patients with no (zero) hospitalizations for CF exacerbations during the study year, zero-inflated Poisson regression models were used to generate incidence rate ratios (IRRs), with 95\% confidence intervals (CIs) for the association between PPI use and number of hospitalizations. This was also used to fit a model examining the association of PPI use, $\mathrm{ppFEV}_{1}$, chronic pseudomonas colonization, homozygous $\Delta \mathrm{F} 508$ genotype and BMI with the number of hospitalizations in the year of study. Covariates for multivariable models were chosen based on clinical reasoning and statistical significance in bivariable analyses. A two-sided P-value of $<0.05$ was used to indicate statistical significance in all analyses. STATA version 13.0 (Statacorp, College Station, TX) was used for statistical analysis.

\section{Results}

Between January and December 2016, 143 patients were seen at the University of Florida Adult Cystic Fibrosis Center and were considered for inclusion. Within this cohort, six patients did not have confirmed CF and 23 did not have 1 year of complete follow-up prior to their index visit and were excluded from the study. A final 114 patients were included in the study (Fig. 1).

Among all study participants, 59 patients (51.8\%) were hospitalized at least once in the study year. Baseline character- 
Table 1. Baseline Characteristics of Patients Hospitalized at Least Once in Year of Study and Non-Hospitalized Patients

\begin{tabular}{llll} 
& $\begin{array}{l}\text { Not hospitalized } \\
\text { in past year }(\mathbf{n}=\mathbf{5 5})\end{array}$ & $\begin{array}{l}\text { Hospitalized at least once } \\
\text { in past year }(\mathbf{n}=\mathbf{5 9})\end{array}$ & P value \\
\hline Male, \% (n) & $54.5 \%(30)$ & $47.5 \%(28)$ & $0.449^{\mathrm{a}}$ \\
Age, median (range) & $30(24-41)$ & $28(24-35)$ & $0.315^{\mathrm{b}}$ \\
BMI, median (range) & $22(20.4-26.5)$ & $20.2(18.3-23.2)$ & $0.003^{\mathrm{b}}$ \\
Homozygous $\Delta$ F508, \% (n) & $39.6 \%(21)$ & $61 \%(36)$ & $0.024^{\mathrm{a}}$ \\
ppFEV1, median (range) & $69 \%(53-81)$ & $40 \%(27-66)$ & $<0.001^{\mathrm{b}}$ \\
Chronic pseudomonas colonization, \% (n) & $65.4 \%(36)$ & $74.6 \%(44)$ & $0.287^{\mathrm{a}}$ \\
GER, \% (n) & $65.4 \%(36)$ & $64.4 \%(38)$ & $0.907^{\mathrm{a}}$ \\
PPI use, \% (n) & $50.9 \%(28)$ & $52.5 \%(31)$ & $0.862^{\mathrm{a}}$ \\
Pancreatic insufficiency, \% (n) & $78.2 \%(43)$ & $83.1 \%(49)$ & $0.510^{\mathrm{a}}$ \\
\hline
\end{tabular}

aPearson's Chi-squared test $\left(X^{2}\right)$. ${ }^{b}$ Mann-Whitney $U$ test.

istics by hospitalization status are shown in Table 1. PPI use was associated with a significantly higher frequency of hospitalization ( 0.9 vs. 1.4 hospitalizations) for pulmonary exacerbation in the study year $(\mathrm{IRR}=1.75, \mathrm{P}=0.009)$. Patients hospitalized at least once in the study year had a significantly lower ppFEV 1 and BMI. A significantly higher percentage of hospitalized patients were chronically colonized with $P$. aeruginosa. There was no significant difference between hospitalized and non-hospitalized patients in rates of GER.

PPI use was common, with 59 (51.8\%) patients having at least 6 months of PPI exposure. Omeprazole was the most commonly used PPI $(\mathrm{n}=25)$, followed by pantoprazole $(\mathrm{n}=$ 18), lansoprazole $(n=12)$ and esomeprazole $(n=4)$. Once daily dosing was observed in $92 \%$ of patients and mean total daily PPI dose was $31.7 \mathrm{mg}( \pm 9.1)$. Baseline characteristics comparing PPI users to non-users are shown in Table 2.

There was no statistically significant difference in number of hospitalizations between patients on high dose PPI therapy (> $40 \mathrm{mg}$ daily) compared to standard dose PPI therapy. The association between PPI exposure and increased frequency of hospitalization persisted in multivariable analysis, factoring in $\mathrm{ppFEV}_{1}$, chronic pseudomonas colonization, BMI and homozygous $\triangle F 508$ genotype. Lower $\mathrm{pFEV}_{1}$ also persisted as a significant predictor of a higher frequency of hospitalization in the multivariable model. The results of the multivariable zeroinflated Poisson regression model are shown in Table 3.

\section{Discussion}

In this retrospective longitudinal study, we examined the association between PPI use and frequency of hospitalizations for pulmonary exacerbations over 1 year in a cohort of adult CF patients. History of PPI use was associated with a higher mean number of hospitalizations over 1 year of observation. Even when controlling for factors traditionally associated with higher frequency hospitalization including lower $\mathrm{pFEV}_{1}$, homozygous $\triangle F 508$ genotype, lower BMI and chronic pseudomonas colonization, exposure to PPI remained independently significantly associated with a higher number of hospitalizations for CF pulmonary exacerbations.

GER is often managed with long term PPI therapy; however, the effects of PPI therapy on pulmonary function and exacerbation in CF patients are unclear. GER itself has been associated with worse pulmonary function in $\mathrm{CF}$ patients. Gustafsson et al were the first to report this association [20], since then multiple studies have demonstrated an association between GER and worse pulmonary function $[11,13,21]$. One

Table 2. Baseline Characteristics of Proton Pump Inhibitor Users as Compared to Non-Users

\begin{tabular}{|c|c|c|c|}
\hline & No daily PPI $(n=55)$ & Daily PPI $(n=59)$ & P value \\
\hline Male, \% (n) & $60 \%(33)$ & $40 \%(25)$ & $0.06^{\mathrm{a}}$ \\
\hline Age, median (range) & $29(24-38)$ & $29(24-34)$ & $0.519^{b}$ \\
\hline F508 homozygous, \% (n) & $55.6(30)$ & $46.6(27)$ & $0.341^{\mathrm{a}}$ \\
\hline $\mathrm{ppFEV}_{1}$, median (range) & $57 \%(37-77)$ & $57 \%(32-75)$ & $0.932^{\mathrm{b}}$ \\
\hline Number of hospitalizations, mean $\pm \mathrm{SD}$ & $0.9 \pm 1.2$ & $1.4 \pm 2.1$ & $0.009^{\mathrm{c}}$ \\
\hline GER, \% (n) & $30.9 \%(17)$ & $96.6 \%(57)$ & $<0.001^{\mathrm{b}}$ \\
\hline Pancreatic insufficiency, \% (n) & $83.6 \%(46)$ & $77.9 \%(46)$ & $0.443^{\mathrm{a}}$ \\
\hline
\end{tabular}

aPearson's Chi-squared test $\left(\mathrm{X}^{2}\right)$. ${ }^{\mathrm{b}}$ Mann-Whitney $\mathrm{U}$ test. ${ }^{\mathrm{C} Z e r o-i n f l a t e d ~ P o i s s o n . ~}$ 
Table 3. Zero-Inflated Poisson Regression Predicting Hospitalization for Pulmonary Exacerbation Over Year of Study

\begin{tabular}{lllllll}
\hline Number of hospitalizations & IRR & Robust standard error & z & P value & 95\% confidence interval \\
\hline PPI use & 1.75 & 0.453 & 2.17 & 0.03 & 1.06 & 2.91 \\
ppFEV $_{1}$ & 0.98 & 0.007 & -2.84 & 0.005 & 0.97 & 0.99 \\
BMI & 1.01 & 0.024 & 0.32 & 0.751 & 0.96 & 1.06 \\
Homozygous $\Delta$ F508 & 1.38 & 0.340 & 1.3 & 0.195 & 0.85 & 2.23 \\
Chronic pseudomonas colonization & 0.63 & 0.203 & -1.44 & 0.149 & 0.33 & 1.18 \\
\hline
\end{tabular}

suggested mechanism is that full esophageal reflux column (extending through the full length of the esophagus) leads to aspiration of gastric contents, ultimately contributing to inflammation and progression of lung disease [22]. In support of this theoretical mechanism is work by Ledson et al who have demonstrated tracheal acidification in adult CF patients suffering from GER [23]. Other suggested mechanisms include increased tracheobronchial mucus secretion secondary to esophageal acidification [12] as well as links between GER and airway neutrophilic inflammation mediated by IL-8 [24].

However, despite this association of GER with worse respiratory function and multiple suggested mechanisms, there is no clear evidence demonstrating a direct causative effect of GER on worse respiratory outcomes [8]. Whether PPI therapy itself plays a role in modifying respiratory outcomes is what we have attempted to explore. The retrospective nature of our study and our small sample size did not allow us to exclude patients with GER, thus whether the increased frequency of hospitalization of patients on PPI therapy demonstrated by our study is confounded by underlying GER is less clear. However, we note that the frequency of GER in both hospitalized and non-hospitalized patients in our study was comparable and not significantly different.

Other studies have attempted to explore the effects of PPI in CF patients. The association between PPI use and worse pulmonary function in CF patients was first reported by Sabati et al in a retrospective study of 201 adult CF patients. This study however was not designed to directly study the effects of PPI use [25]. Later, in a prospective study of CF patients randomized to placebo or esomeprazole, DiMango et al showed a trend towards earlier and more frequent exacerbations in patients randomized to PPI therapy [22]. Work by Pauwels et al offers a biological explanation to the above clinical findings; they demonstrated that gastric juice under the effects of PPI had a significantly enhanced inflammatory effect on CF bronchial epithelial cells as compared to gastric juice not affected by PPI [26]. This leads the authors to believe that PPI therapy in CF patients may result in paradoxically increased inflammatory effects on the airway. In non-CF patients, multiple studies have demonstrated an association between PPI use and CAP in both the inpatient and outpatient settings [16, 27, 28]. This association demonstrated a dose-response relationship as shown by Laheij et al [16]. In our study, the small sample size and the relatively small number of patients on high dose therapy limited our power to study such an effect.

Inappropriate PPI use is well-documented in non-CF populations [29-31]. This same issue appears to persist in the CF population, where classic adverse events associated with PPI therapy such as bone fractures [32] and B12 deficiency [33] are likely to have a higher impact. More than half of our patient cohort $(51.7 \%)$ had a history of PPI therapy. This is in line with other studies of CF patients; a study of practice patterns at 29 French CF centers by Languepin et al illustrated a liberal pattern of PPI use in CF patients. The authors found that all CF centers relied on long-term PPI as first-line therapy for GER, while no center carried out routine diagnostic evaluation, nor any testing for efficacy of treatment after initiation of therapy [9].

Our study has several limitations. The retrospective nature of our study allows only determination of association and does not support a cause-effect relationship of PPI therapy on pulmonary exacerbations. The inclusion of patients who were receiving PPI therapy for GER is a possible confounder for our results; however, the frequency of patients with GER was similar in both hospitalized and non-hospitalized patients. Finally, this is a single-center study with a relatively small sample size and this possibly limits generalizability; however, given the low prevalence of CF, we believe our sample size was adequate. Improving our generalizability is that our sample encompassed a wide spectrum of ages and disease severity as well as the fact that the University of Florida Adult CF Center is a major referral center for patients in the southeastern United States.

In conclusion, the results from our study demonstrate a significant association between PPI use and higher mean number of hospitalizations for CF pulmonary exacerbations. Larger, prospective trials are warranted to further clarify the effects of PPI therapy on pulmonary function in CF patients. Prescribers of PPI therapy should exercise pharmacovigilance; frequently re-evaluating indications and appropriateness of therapy and in the setting of GER considering alternate management modalities such as anti-reflux surgery where appropriate.

\section{Disclosures}

This research was not supported by any grant, internal or external funding. The authors declare no financial arrangements or conflict of interest related to the research or assistance with manuscript preparation.

\section{References}

1. O'Sullivan BP, Freedman SD. Cystic fibrosis. Lancet. 2009;373(9678):1891-1904.

2. Ferkol T, Rosenfeld M, Milla CE. Cystic fibrosis pulmo- 
nary exacerbations. J Pediatr. 2006;148(2):259-264.

3. Goss CH, Burns JL. Exacerbations in cystic fibrosis. 1: Epidemiology and pathogenesis. Thorax. 2007;62(4):360367.

4. Cogen JD, Oron AP, Gibson RL, Hoffman LR, Kronman MP, Ong T, Rosenfeld M. Characterization of Inpatient Cystic Fibrosis Pulmonary Exacerbations. Pediatrics. 2017;139(2).

5. Sanders DB, Bittner RC, Rosenfeld M, Hoffman LR, Redding GJ, Goss $\mathrm{CH}$. Failure to recover to baseline pulmonary function after cystic fibrosis pulmonary exacerbation. Am J Respir Crit Care Med. 2010;182(5):627632.

6. Britto MT, Kotagal UR, Hornung RW, Atherton HD, Tsevat J, Wilmott RW. Impact of recent pulmonary exacerbations on quality of life in patients with cystic fibrosis. Chest. 2002;121(1):64-72.

7. Liou TG, Adler FR, Fitzsimmons SC, Cahill BC, Hibbs JR, Marshall BC. Predictive 5-year survivorship model of cystic fibrosis. Am J Epidemiol. 2001;153(4):345-352.

8. Robinson NB, DiMango E. Prevalence of gastroesophageal reflux in cystic fibrosis and implications for lung disease. Ann Am Thorac Soc. 2014;11(6):964-968.

9. Languepin J, Munck A, Gros G, Mas E. 194 A French survey on gastro-oesophageal reflux in CF: need for guidance. J Cyst Fibros. 2014;13:S96.

10. Stringer DA, Sprigg A, Juodis E, Corey M, Daneman A, Levison HJ, Durie PR. The association of cystic fibrosis, gastroesophageal reflux, and reduced pulmonary function. Can Assoc Radiol J. 1988;39(2):100-102.

11. Button BM, Roberts S, Kotsimbos TC, Levvey BJ, Williams TJ, Bailey M, Snell GI, et al. Gastroesophageal reflux (symptomatic and silent): a potentially significant problem in patients with cystic fibrosis before and after lung transplantation. J Heart Lung Transplant. 2005;24(10):1522-1529.

12. Blondeau K, Dupont LJ, Mertens V, Verleden G, Malfroot A, Vandenplas Y, Hauser B, et al. Gastro-oesophageal reflux and aspiration of gastric contents in adult patients with cystic fibrosis. Gut. 2008;57(8):1049-1055.

13. Palm K, Sawicki G, Rosen R. The impact of reflux burden on Pseudomonas positivity in children with cystic fibrosis. Pediatr Pulmonol. 2012;47(6):582-587.

14. Canani RB, Cirillo P, Roggero P, Romano C, Malamisura B, Terrin G, Passariello A, et al. Therapy with gastric acidity inhibitors increases the risk of acute gastroenteritis and community-acquired pneumonia in children. Pediatrics. 2006;117(5):e817-820.

15. Lambert AA, Lam JO, Paik JJ, Ugarte-Gil C, Drummond MB, Crowell TA. Risk of community-acquired pneumonia with outpatient proton-pump inhibitor therapy: a systematic review and meta-analysis. PLoS One. 2015;10(6):e0128004.

16. Laheij RJ, Sturkenboom MC, Hassing RJ, Dieleman J, Stricker BH, Jansen JB. Risk of community-acquired pneumonia and use of gastric acid-suppressive drugs. JAMA. 2004;292(16):1955-1960.

17. de Jager CP, Wever PC, Gemen EF, van Oijen MG, van Gageldonk-Lafeber AB, Siersema PD, Kusters GC, et al.
Proton pump inhibitor therapy predisposes to community-acquired Streptococcus pneumoniae pneumonia. Aliment Pharmacol Ther. 2012;36(10):941-949.

18. Canton R, Cobos N, de Gracia J, Baquero F, Honorato J, Gartner S, Alvarez A, et al. Antimicrobial therapy for pulmonary pathogenic colonisation and infection by Pseudomonas aeruginosa in cystic fibrosis patients. Clin Microbiol Infect. 2005;11(9):690-703.

19. O’Sullivan BP, Baker D, Leung KG, Reed G, Baker $\mathrm{SS}$, Borowitz D. Evolution of pancreatic function during the first year in infants with cystic fibrosis. J Pediatr. 2013;162(4):808-812 e801.

20. Gustafsson PM, Fransson SG, Kjellman NI, Tibbling L. Gastro-oesophageal reflux and severity of pulmonary disease in cystic fibrosis. Scand J Gastroenterol. 1991;26(5):449-456.

21. Navarro J, Rainisio M, Harms HK, Hodson ME, Koch C, Mastella G, Strandvik B, et al. Factors associated with poor pulmonary function: cross-sectional analysis of data from the ERCF. European Epidemiologic Registry of Cystic Fibrosis. Eur Respir J. 2001;18(2):298-305.

22. Dimango E, Walker P, Keating C, Berdella M, Robinson N, Langfelder-Schwind E, Levy D, et al. Effect of esomeprazole versus placebo on pulmonary exacerbations in cystic fibrosis. BMC Pulm Med. 2014;14:21.

23. Ledson MJ, Wilson GE, Tran J, Walshaw MJ. Tracheal microaspiration in adult cystic fibrosis. J R Soc Med. 1998;91(1):10-12.

24. Sacco O, Silvestri M, Sabatini F, Sale R, Moscato G, Pignatti P, Mattioli G, et al. IL-8 and airway neutrophilia in children with gastroesophageal reflux and asthma-like symptoms. Respir Med. 2006;100(2):307-315.

25. Sabati AA, Kempainen RR, Milla CE, Ireland $M$, Schwarzenberg SJ, Dunitz JM, Khan KM. Characteristics of gastroesophageal reflux in adults with cystic fibrosis. J Cyst Fibros. 2010;9(5):365-370.

26. Pauwels A, Verleden S, Farre R, Vanaudenaerde BM, Van Raemdonck D, Verleden G, Sifrim D, et al. The effect of gastric juice on interleukin- 8 production by cystic fibrosis primary bronchial epithelial cells. J Cyst Fibros. 2013;12(6):700-705.

27. Gulmez SE, Holm A, Frederiksen H, Jensen TG, Pedersen C, Hallas J. Use of proton pump inhibitors and the risk of community-acquired pneumonia: a population-based case-control study. Arch Intern Med. 2007;167(9):950955.

28. Eurich DT, Sadowski CA, Simpson SH, Marrie TJ, Majumdar SR. Recurrent community-acquired pneumonia in patients starting acid-suppressing drugs. Am J Med. 2010;123(1):47-53.

29. Heidelbaugh JJ, Goldberg KL, Inadomi JM. Magnitude and economic effect of overuse of antisecretory therapy in the ambulatory care setting. Am J Manag Care. 2010;16(9):e228-234.

30. Perwaiz MK, Posner G, Hammoudeh F, Schmidt F, Neupane N, Enriquez D, Gulati N. Inappropriate use of intravenous PPI for stress ulcer prophylaxis in an inner city community hospital. J Clin Med Res. 2010;2(5):215-219.

31. Leri F, Ayzenberg M, Voyce SJ, Klein A, Hartz L, Smego 
RA, Jr. Four-year trends of inappropriate proton pump inhibitor use after hospital discharge. South Med J. 2013;106(4):270-273.

32. Gray SL, LaCroix AZ, Larson J, Robbins J, Cauley JA, Manson JE, Chen Z. Proton pump inhibitor use, hip fracture, and change in bone mineral density in postmenopau- sal women: results from the Women's Health Initiative. Arch Intern Med. 2010;170(9):765-771.

33. Valuck RJ, Ruscin JM. A case-control study on adverse effects: $\mathrm{H} 2$ blocker or proton pump inhibitor use and risk of vitamin B12 deficiency in older adults. J Clin Epidemiol. 2004;57(4):422-428. 